December 1992

\title{
MODULAR STRUCTURE AND DUALITY IN CONFORMAL QUANTUM FIELD THEORY
}

\author{
R. Brunetti ${ }^{1 *} \bullet$, D. Guido ${ }^{2 *}$ and R. Longo ${ }^{2 *}$ \\ (1) Dipartimento di Fisica, Università di Napoli "Federico II" \\ Mostra d'Oltremare, Pad. 19 I-80125, Napoli, Italy \\ E-mail BRUNETTI@NAPOLI.INFN.IT \\ $(2)$ Dipartimento di Matematica, Università di Roma "Tor Vergata" \\ Via della Ricerca Scientifica I-00133, Roma, Italy. \\ E-mail GUIDO@MAT.UTOVRM.IT, LONGO@MAT.UTOVRM.IT
}

\begin{abstract}
Making use of a recent result of Borchers, an algebraic version of the BisognanoWichmann theorem is given for conformal quantum field theories, i.e. the Tomita-Takesaki modular group associated with the von Neumann algebra of a wedge region and the vacuum vector concides with the evolution given by the rescaled pure Lorentz transformations preserving the wedge. A similar geometric description is valid for the algebras associated with double cones. Moreover essential duality holds on the Minkowski space $M$, and Haag duality for double cones holds provided the net of local algebras is extended to a pre-cosheaf on the superworld $\tilde{M}$, i.e. the universal covering of the Dirac-Weyl compactification of $M$. As a consequence a PCT symmetry exists for any algebraic conformal field theory in even spacetime dimension. Analogous results hold for a Poincaré covariant theory provided the modular groups corresponding to wedge algebras have the expected geometrical meaning and the split property is satisfied. In particular the Poincaré representation is unique in this case.
\end{abstract}

* Supported in part by Ministero della Ricerca Scientifica and CNR-GNAFA.

- Supported in part by INFN, sez. Napoli. 


\section{Introduction}

Haag duality in Quantum Field Theory is the property that local observable algebras maximally obeys the causality principle: if $\mathcal{R}(\mathcal{O})$ is the von Neumann algebra of the observables localized in the double cone $\mathcal{O}$ of the Minkowski space $M$, then $\mathcal{R}(\mathcal{O})$ is the commutant of the von Neumann algebra $\mathcal{R}\left(\mathcal{O}^{\prime}\right)$ of the observables localized in the space-like complement $\mathcal{O}^{\prime}$ of $\mathcal{O}$

$$
\mathcal{R}\left(\mathcal{O}^{\prime}\right)=\mathcal{R}(\mathcal{O})^{\prime}
$$

Duality plays an important role in the structural analysis of algebraic Quantum Field Theory [9] and has long been verified in free field models [1]. If the local algebras are generated by Wightman fields, Bisognano and Wichmann [2] have shown the general result that duality holds for the von Neumann algebras associated with wedge shaped regions $W$, namely

$$
\mathcal{R}\left(W^{\prime}\right)=\mathcal{R}(W)^{\prime}
$$

where $W$ is any Poincaré transformed of the region $\left\{x \in M\left|x_{1}>\right| x_{0} \mid\right\}$. This property is called essential duality since it allows to enlarge the original observable algebras of double cones so that Haag duality holds true. Their basic result is obtained by the computation of the Tomita-Takesaki modular operator $\Delta_{W}$ associated to $\mathcal{R}(W)$ with respect to the vacuum vector $\Omega$ [20], the latter being cyclic and separating because of the Reeh-Schlieder theorem. In this case the modular group is the (rescaled) oneparameter group of pure Lorentz transformations leaving $W$ invariant and the modular conjugation $J_{W}$ is the product of the PCT symmetry and a rotation; the essential duality then follows at once by Tomita's commutation theorem

$$
\mathcal{R}(W)^{\prime}=J_{W} \mathcal{R}(W) J_{W}=\mathcal{R}\left(W^{\prime}\right) .
$$

This identification of the modular group has several interesting consequences; beside duality, we mention here, as an aside, the relation with the Hawking effect, see [9], due to the KMS (temperature) condition characterizing this evolution, and the Poincaré covariance of the superselection sectors with finite statistics in [14].

One should not expect a sharp geometrical description for the modular group of the algebra $\mathcal{R}(\mathcal{O})$ of a double cone $\mathcal{O}$, since in general there are not enough space-time symmetries that preserve $\mathcal{O}$; however, as a consequence of the Bisognano-Wichmann theorem, the modular group of $\mathcal{R}(\mathcal{O})$ has a geometrical meaning in a conformally invariant theory [15].

The purpose of the present work is to provide an intrinsic, Wightman field independent, algebraic derivation of the Bisognano-Wichmann theorem in the case of a conformally invariant theory. 
We were motivated by a recent general result of Borchers [3] showing that part of the geometric behavior of $\Delta_{W}$ follows automatically from the positivity of the energymomentum operators: $\Delta_{W}$ has the expected commutation relations with the translation operators. This result however does not furnish the commutation relations between modular operators associated with different wedges and, in space-time dimension greater than two, does not provide the Bisognano-Wichmann identification of the modular automorphism group of $\mathcal{R}(W)$, indeed simple counter-examples illustrate how it may be violated in general.

We shall show that essential duality holds automatically in a conformal theory of any space-time dimension. But Haag duality for double cones fails in general because the local algebras actually live in a superworld $\tilde{M}[19,17]$, a $\infty$-sheeted cover of a compactified Minkowski space. However there is a natural procedure to extend the original net of local algebras to a causal pre-cosheaf of von Neumann algebras (i.e. an inclusion preserving map $\mathcal{O} \rightarrow \mathcal{R}(\mathcal{O})$ ) on $\tilde{M}$, and Haag duality holds there.

As a further consequence, we shall show that an algebraic conformal theory admits automatically a PCT symmetry [21].

In particular for a Möbius covariant pre-cosheaf of local algebras on $S^{1}$, duality holds on $S^{1}$ without any assumption other than positivity of the energy. Nevertheless duality fails in general on the cut circle $\simeq \mathbf{R}$. This phenomenon, discussed in [6] in this specialization, is already present in [15] concerning the time-like duality; we shall reformulate the examples in [15] to get models of conformal theories on $S^{1}$ with the desired properties.

Our results parallel an independent work of Fredenhagen and Jörss [11] where similar results are obtained by a different route: they construct Wightman fields associated with an algebraic conformal field theory on $S^{1}$, under a finite multiplicity condition for the Möbius representation.

Part of our analysis extends to the general case of Poincaré covariant nets. Assuming that the modular group of the von Neumann algebra of any given wedge region has a geometrical meaning, we can show that the Bisognano-Wichmann interpretation holds, provided the net fulfills the split property. The latter condition is indeed necessary and guarantees the uniqueness of the Poincaré covariant action.

After our work was completed we received a preprint of Gabbiani and Fröhlich [12] that contains similar analysis for algebraic conformal field theories on $S^{1}$. 


\section{Algebraic Conformal Quantum Field Theory, general set- ting.}

In this section we describe a Conformal Quantum Field Theory in the algebraic approach. Our aim is to give a self-contained introduction in our setting of known features.

Geometrical preliminaries. In the following we consider a Lie group $G$ acting by local diffeomorphisms on a manifold $M$, i.e. there exists an open set $W \subset G \times M$ and a $C^{\infty}$ map

$$
\begin{aligned}
& T: \quad W \quad \rightarrow \quad M \\
& (g, x) \mapsto T_{g} x
\end{aligned}
$$

with the following properties:

(i) $\forall x \in M, V_{x} \equiv\{g \in G:(g, x) \in W\}$ is an open connected neighborhood of the identity $e \in G$

(ii) $T_{e} x=x, \forall x \in M$

(iii) If $(g, x) \in W$, then $V_{T_{g} x}=V_{x} g^{-1}$ and moreover for any $h \in G$ such that $h g \in V_{x}$

$$
T_{h} T_{g} x=T_{h g} x
$$

We say that a local action of a Lie group $G$ on a manifold $\mathrm{M}$ is quasi-global if the open set

$$
\{x \in M:(g, x) \in W\}
$$

is the complement of a meager set $S_{g}$, and the following equation holds:

$$
\lim _{x \rightarrow x_{0}} T_{g} x=+\infty, \quad g \in G, \quad x_{0} \in S_{g}
$$

where $x$ approaches $x_{0}$ out of $S_{g}$ and a point goes to infinity when it is eventually out of any compact subset of $M$.

1.1 Proposition. If $T$ is a transitive quasi-global action of $G$ on $M$, then there exists a unique "T-completion" of $M$, i.e. a manifold $\bar{M}$ such that $M$ is a dense open subset of $\bar{M}$ and the action $T$ extends to a transitive global action on $\bar{M}$.

Proof Let $\mathcal{E}$ be the space of the continuous bounded functions $\varphi$ on $M$ such that, for any given $g \in G$, the function $T_{g}^{*} \varphi$ defined by

$$
T_{g}^{*} \varphi(x) \equiv \varphi\left(T_{g} x\right), \quad x \notin S_{g}
$$

has a continuous extension to $M$. 
Since a countable union of meager sets is meager, it is easy to see that $\mathcal{E}$ is a $C^{*}$-algebra, and $g \rightarrow T_{g}^{*}$ is an action of $G$ by isomorphisms of $\mathcal{E}$.

Moreover $\mathcal{E}$ contains the algebra $C_{0}(M)$ of continuous functions on $M$ vanishing at infinity because, by condition (1.2),

$$
T_{g}^{*} \varphi(x) \equiv \begin{cases}\varphi\left(T_{g} x\right) & \text { if } x \notin S_{g} \\ 0 & \text { if } x \in S_{g}\end{cases}
$$

is a continuous function, for any $\varphi \in C_{0}(M)$ and for any fixed $g \in G$. We denote by $C_{G}$ the minimal $C^{*}$-subalgebra of $\mathcal{E}$ containing $C_{0}(M)$ and globally invariant under the action $T^{*}$.

We show that the spectrum $\bar{M}$ of $C_{G}$ is the requested completion, where the (global) action of $G$ on $\bar{M}$ is given by

$$
T_{g} p=p \cdot T_{g}^{*}, \quad p \in \bar{M} .
$$

We notice that $C_{G}$ does not necessarily contain the identity, therefore $\bar{M}$ is not compact in general. Moreover, by well known arguments [13], the spectrum of the $C^{*}$-algebra obtained adding the identity to $C_{G}$ is a compactification of $M$ and is the one-point compactification of $\bar{M}$, hence the natural embedding $M \hookrightarrow \bar{M}$ is dense.

The transitivity of the action on $\bar{M}$ follows by the minimal choice of $C_{G}$. In fact, let us consider the orbit in $\bar{M}$ containing $M$,

$$
\bar{M}_{0} \equiv\left\{T_{g} x: g \in G\right\}
$$

where $x$ is a point of $M$ as a subspace of $\bar{M}$. By the transitivity on $M, \bar{M}_{0}$ does not depend on $x$ and the action of $G$ is transitive on $\bar{M}_{0}$. We have natural embeddings

$$
C_{0}(M) \subseteq C_{0}\left(\bar{M}_{0}\right) \subseteq C_{G}
$$

and $C_{0}\left(\bar{M}_{0}\right)$ is globally invariant under $T^{*}$. Since $C_{G}$ is, by construction, the minimal $C^{*}$-algebra with this properties, then $\bar{M}_{0}=\bar{M}$.

By minimality, any other $T$-completion of $M$ contains $\bar{M}$, and therefore the transitivity requirement implies uniqueness.

Finally, $\bar{M}$ is a homogeneous space for the Lie group $G$, hence it is a $C^{\infty}$ manifold.

1.2 Proposition. In the hypotheses of the previous proposition, we consider the universal covering $\tilde{G}$ of $G$ and the universal covering $\tilde{M}$ of $\bar{M}$. Then the action $T$ lifts to a transitive global action $\tilde{T}$ of $\tilde{G}$ on $\tilde{M}$.

Proof The group $\tilde{G}$ has a canonical global transitive action on $\bar{M}$, i.e. $T \cdot \pi$ where $\pi: \tilde{G} \rightarrow G$ is the covering map. The identity component of the isotropy group of a point $\left(\tilde{G}_{x}\right)_{0}$ is a connected closed subgroup of $\tilde{G}$, therefore [18], the manifold

$$
\tilde{M} \equiv \tilde{G} /\left(\tilde{G}_{x}\right)_{0}
$$


is simply connected and is a covering of $\bar{M}$, i.e. it is the universal covering of $\bar{M}$. Then $\tilde{M}$ is a homogeneous space for $\tilde{G}$, and the thesis follows.

Conformal action on the Minkowski space. In the following we specialize the preceding analysis to the action of the conformal group on the Minkowski space $M$. The action of the conformal group $\mathcal{C}$ on a point of $M$ (resp. of the universal covering $\tilde{\mathcal{C}}$ on $\tilde{M}$ ) will be denoted by $x \rightarrow g x$. As is known, $M$ is the manifold $\mathbf{R}^{d}$ with constant pseudo-metric tensor $q$ with signature

$$
+1, \underbrace{-1, \ldots,-1}_{(\mathrm{d}-1)-\text { times }} .
$$

The corresponding Minkowski norm of a vector $x \in M$ is

$$
x^{2}=x_{0}^{2}-x_{1}^{2}-\ldots-x_{d-1}^{2} .
$$

When $d>2$ the conformal group consists of the local diffeomorphisms $\varphi$ of $M$ which preserve the pseudo-metric tensor $q$ up to a non-vanishing function $\mu$ :

$$
\varphi^{*} q=\mu q
$$

This group is a $\frac{(d+2)(d+1)}{2}$ dimensional Lie group, and the Lie algebra of its identity component $\mathcal{C}$ is generated (as a vector space) by the following objects:

$\begin{array}{ll}\text { translations } & d \text { generators } \\ \text { boosts } & d-1 \text { generators } \\ \text { rotations } & \frac{(d-1)(d-2)}{2} \text { generators } \\ \text { dilations } & 1 \text { generator } \\ \text { special transformations } & d \text { generators }\end{array}$

The special transformations are the elements of the form

$$
\rho \tau_{a} \rho
$$

where $\tau_{a}, a \in \mathbf{R}^{d}$, is a translation and $\rho$ is the relativistic ray inversion:

$$
\rho x=-\frac{x}{x^{2}} \quad x^{2} \neq 0 .
$$

When $d \leq 2$ the conformal group $\mathcal{C}$ is, by definition, the one generated by the transformations described in (1.4).

The Conformal Universe. The conformal group $\mathcal{C}$ acts quasi-globally on $M$, and therefore the completion $\bar{M}$ is defined. 
For convenience of the reader, we follow [22] and give the more explicit construction of $\bar{M}$ by Dirac and Weyl $[7,23]$. Given $\mathbf{R}^{d+2}$ with signature

$$
+1, \underbrace{-1, \ldots,-1}_{d-\text { times }}+1
$$

we consider the manifold $N$ whose points are the isotropic rays of the light cone, i.e.

$$
N=\left\{\left(\xi_{0}, \ldots \xi_{n+1}\right) \in \mathbf{R}^{d+2} \backslash\{0\}:+\xi_{0}^{2}-\xi_{1}^{2}-\ldots-\xi_{d}^{2}+\xi_{d+1}^{2}=0\right\} / \mathbf{R}^{*}
$$

where $\mathbf{R}^{*}=\mathbf{R} \backslash\{0\}$ acts by multiplication on $\mathbf{R}^{d+2}$. The Lie group $\operatorname{PSO}(d, 2)$ acts transitively on this manifold by global diffeomorphisms, and it is easy to check that the map $M \rightarrow N$ given by

$$
\left\{\begin{aligned}
\xi_{i} & =x_{i} \quad i<d \\
\xi_{d} & =\frac{1-x^{2}}{2} \\
\xi_{d+1} & =\frac{1+x^{2}}{2}
\end{aligned}\right.
$$

is a dense embedding such that the restriction of the action of $P S O(d, 2)$ to $M$ corresponds to the conformal transformations. The uniqueness proven in Proposition 1.2 shows that $N=\bar{M}$.

The Dirac-Weyl description shows $\bar{M}$ to be a compact manifold diffeomorphic to $\left(S^{d-1} \times S^{1}\right) / \mathbf{Z}_{2}$. When $d>2$, the universal covering $\tilde{M}$ is infinite sheeted and is diffeomorphic to $\left(S^{d-1} \times \mathbf{R}\right)$. The map described in equation (1.5) lifts to a natural embedding of $M$ into $\tilde{M}$, and the covering map from $\tilde{M}$ to $\bar{M}$ shows that $\tilde{M}$ contains infinitely many copies of $M$ as submanifolds.

One of the main advantages of dealing with the manifold $\tilde{M}$ instead of $\bar{M}$ is that a global causal structure is naturally defined on it, i.e. the time ordering gives rise to a global ordering relation which extends the ordering on $M$, and a notion of (nonpositive definite) geodesic distance is also well-defined, and is locally equivalent to the one in $M$. As a consequence each point divides $\tilde{M}$ in three parts, the relative future, i.e. the points at time-like distance which follow the point, the relative past, i.e. the points at time-like distance which precede the point, and the relative present, i.e. the points at space-like (or light-like) distance from the point. As we shall see, conformal quantum field theories live naturally on $\tilde{M}$.

When $d \leq 2, \bar{M}$ is diffeomorphic to $\left(S^{1}\right)^{d}$, and therefore its universal covering is $\mathbf{R}^{d}$, but this manifold is rather unphysical. In fact we may find two space-like separated embeddings of $M$ in $\mathbf{R}^{d}$. Therefore we use the convention $\tilde{M} \equiv S^{1} \times \mathbf{R}$ when $d=2$, and $\tilde{M} \equiv S^{1}$ when $d=1$. 
We also mention that when $d$ is odd $(d \neq 1)$ the manifold $\bar{M}$ is not orientable, and physical theories live on orientable coverings of $\bar{M}$ (cf. Proposition 1.7 and the odd-dimensional examples at the end of Section 2).

In the following we shall consider the family $\tilde{\mathcal{K}}$ of the subregions of $\tilde{M}$ which are images of double cones in $M$ under conformal transformations in $\tilde{\mathcal{C}}$. We notice that all double cones, wedges and light-cones of $M$ belong to the family $\tilde{\mathcal{K}}$, see e.g. [15]. Now we list some properties of $\tilde{\mathcal{K}}$ :

\subsection{Proposition.}

(i) All elements of $\tilde{\mathcal{K}}$ are open contractible precompact submanifolds of $\tilde{M}$. They are a fundamental set of neighborhoods for $\tilde{M}$.

(ii) $\tilde{\mathcal{C}}$ acts "transitively" on $\tilde{\mathcal{K}}$, i.e.

$$
\forall \mathcal{O}_{1}, \mathcal{O}_{2} \in \tilde{\mathcal{K}} \quad \exists g \in \tilde{\mathcal{C}}: g \mathcal{O}_{1}=\mathcal{O}_{2}
$$

(iii) The identity component $\tilde{\mathcal{C}}(\mathcal{O})_{0}$ of the group of the conformal diffeomorphisms that preserve $\mathcal{O}$,

$$
\tilde{\mathcal{C}}(\mathcal{O})=\{g \in \tilde{\mathcal{C}}: g \mathcal{O}=\mathcal{O}\}
$$

acts transitively on $\mathcal{O}, \mathcal{O} \in \tilde{\mathcal{K}}$.

(iv) The space-like complement $\mathcal{O}^{\prime}$ of a region $\mathcal{O} \in \tilde{\mathcal{K}}$ belong to $\tilde{\mathcal{K}}$.

$(v)$ The family $\tilde{\mathcal{K}}$ is not a net, in fact the union of a region and of its causal complement is not contained in any region of $\tilde{\mathcal{K}}$.

Proof Immediate.

With each region $\mathcal{O} \in \tilde{\mathcal{K}}$, we shall associate a one-parameter group $\Lambda_{t}^{\mathcal{O}}$ of conformal transformations which preserve $\mathcal{O}$ and commute with all $\mathcal{O}$-preserving conformal transformations:

$$
\mathcal{O} \in \tilde{\mathcal{K}} \rightarrow\left\{\Lambda_{t}^{\mathcal{O}}, \quad t \in \mathbf{R}\right\} \subset \tilde{\mathcal{C}}
$$

These groups will have the following coherence property:

$$
\Lambda_{t}^{\mathcal{O}_{2}}=g^{-1} \Lambda_{t}^{\mathcal{O}_{1}} g, \quad \mathcal{O}_{2}=g \mathcal{O}_{1}, \mathcal{O}_{i} \in \mathcal{K} \quad g \in \tilde{\mathcal{C}}
$$

Therefore, by Proposition 1.3(ii), they will be completely determined if we assign $\Lambda_{t}^{\mathcal{O}}$ for one region $\mathcal{O}$. For reader's convenience, we describe explicitly the conformal transformations for three particular regions in $M$ (cf. $[15,4]$ ).

The wedge $W_{1}$ :

$$
W_{1}=\left\{x \in M: x_{1}>\left|x_{0}\right|\right\} .
$$


The group $\Lambda_{t}^{W_{1}}$ is the one-parameter group of pure Lorentz transformations (boosts) along the $x_{1}$ axis; its action on $\left(x_{0}, x_{1}\right)$ is given by the matrices

$$
\left(\begin{array}{cc}
\cosh 2 \pi t & -\sinh 2 \pi t \\
-\sinh 2 \pi t & \cosh 2 \pi t
\end{array}\right)
$$

The double cone $\mathcal{O}_{1}$ :

$$
\mathcal{O}_{1}=\left\{x \in M:\left|x_{0}\right|+|\vec{x}|<1\right\}
$$

The group $\Lambda_{t}^{\mathcal{O}_{1}}$ commutes with the rotations, hence is determined by its action on the $\left(x_{0}, x_{1}\right)$-plane:

$$
\Lambda_{t}^{\mathcal{O}_{1}} x_{ \pm}=\frac{\left(1+x_{ \pm}\right)-e^{-2 \pi t}\left(1-x_{ \pm}\right)}{\left(1+x_{ \pm}\right)-e^{-2 \pi t}\left(1+x_{ \pm}\right)}
$$

where we posed $x_{ \pm}=x_{0} \pm x_{1}$.

The future cone $V_{+}$:

$$
V_{+}=\left\{x \in M: x_{0}>0, x^{2}>0\right\}
$$

The one-parameter group $\Lambda_{t}^{V_{+}}$is the dilation subgroup:

$$
\Lambda_{t}^{V_{+}}=D\left(e^{t}\right)
$$

The universal covering $\tilde{\mathcal{C}} \quad \quad$ The universal covering $\tilde{\mathcal{C}}$ of the conformal group $\mathcal{C}$ turns out to be a central extension of $\mathcal{C}$ with fiber $\mathbf{Z} \times \mathbf{Z}_{2}$. The $\mathbf{Z}_{2}$ component acts trivially on $\tilde{M}$ and $\tilde{\mathcal{C}} / \mathbf{Z}_{2}$ acts effectively on it (see e.g. [22]).

Now we prove some simple properties on the conformal group we shall need in Section 2 .

1.4 Proposition. The groups $\mathcal{C}$ and $\tilde{\mathcal{C}}$ are perfect groups, i.e. they coincide with their commutator subgroups.

Proof Since both $\mathcal{C}$ and $\tilde{\mathcal{C}}$ are semi-simple Lie groups, the result follows by the observations in [18], p. 345.

1.5 Proposition. The conformal transformations $R_{i}, i=1, \ldots, d-1$ given by

$$
R_{i} x=-\frac{1}{x^{2}}\left(x_{0}, \ldots x_{i-1},-x_{i}, x_{i+1}, \ldots, x_{d-1}\right)
$$

are in the identity component of $\mathcal{C}$. Each $R_{i}$ has only two liftings $\pm R_{i}$ of order 4 in $\tilde{\mathcal{C}}$.

Proof (Sketch) We observe that

$$
U(\alpha) \equiv \tau_{i}(-\cot \alpha) D\left((\sin \alpha)^{-2}\right) R_{i} \tau_{i}(-\cot \alpha)
$$


is a one-parameter subgroup of $\mathcal{C}$, where $\tau_{i}(\cdot)$ are the translations along the $i-t h$ axis. Moreover $U(\alpha)$ satisfies

$$
\left\{\begin{array}{c}
U(0)=U(\pi)=e \\
U\left(\frac{\pi}{2}\right)=R_{i}
\end{array}\right.
$$

hence the first assertion follows. Lifting this subgroup to $\tilde{U}(\alpha) \in \tilde{\mathcal{C}}$ we get a group of period $2 \pi$, therefore $\pm R_{i} \equiv \tilde{U}\left( \pm \frac{\pi}{2}\right)$ are the requested liftings. Since the fiber of the covering $\tilde{\mathcal{C}} \rightarrow \mathcal{C}$ is $\mathbf{Z} \times \mathbf{Z}_{2}$, any other lifting has infinite order.

1.6 Proposition. For each $i=1, \ldots, d-1$, the translation subgroup and $R_{i}$ generate the conformal group $\mathcal{C}$. The same result holds in $\tilde{\mathcal{C}}$ when $R_{i}$ is replaced by any of its liftings $\pm R_{i}$ (cf. [22] for an analogous statement in the 4-dimensional case).

Proof (Sketch) A straightforward calculation shows that the equation

$$
\tau_{i}(a) R_{i} \tau_{i}(1 / a) R_{i} \tau_{i}(a) R_{i}=D\left(a^{2}\right)
$$

holds in $\mathcal{C}$. Then, since dilations are the transformations $\Lambda^{V_{+}}$for the future light cone and such a cone can be mapped into any wedge by a suitable product of translations and $R_{i}$, boosts are in the group generated by $\tau$ and $R_{i}$ [15]. The first statement follows because boosts and translations generate the Poincaré group and, together with $R_{i}$, the conformal group $\mathcal{C}(\mathrm{cf}$. formulas $(1.4)$ ). The statement for $\tilde{\mathcal{C}}$ is proven in a similar way.

1.7 Proposition. When $d$ is odd, the change of sign of a space coordinate $P_{i}$ is in the identity component of $\mathcal{C}$. As a consequence $\bar{M}$ is not orientable.

Proof The first part of the Proposition follows from straightforward calculations similar to those in Propositions 1.5 and 1.6. The rest follows because the Jacobian of $P_{i}$ is negative.

Conformal Quantum Field Theories. A local Conformal Quantum Field Theory in dimension $d$ is described by a causal additive pre-cosheaf of von Neumann algebras on the double cones of $M=\mathbf{R}^{d}$ with Minkowski structure, i.e. a map

$$
\mathcal{A}: \mathcal{O} \rightarrow \mathcal{A}(O), \quad \mathcal{O} \in \mathcal{K}
$$


where $\mathcal{K}$ is the family of the double cones in $M$, such that

$$
\begin{array}{rlrl}
\mathcal{O}_{1} \subset \mathcal{O}_{2} & \Rightarrow \mathcal{A}\left(\mathcal{O}_{1}\right) \subset \mathcal{A}\left(\mathcal{O}_{2}\right) & \\
\mathcal{A}(\mathcal{O}) & \subset \mathcal{A}\left(\mathcal{O}^{\prime}\right)^{\prime} & & \text { (causality) } \\
\mathcal{A}\left(\cup_{n} \mathcal{O}_{n}\right) & =\vee_{n} \mathcal{A}\left(\mathcal{O}_{n}\right) & & \text { (additivity) }
\end{array}
$$

where $\mathcal{O}^{\prime}$ is the space-like complement of $\mathcal{O}$ and the regions $\mathcal{O}, \mathcal{O}_{n}$ and $\cup \mathcal{O}_{n}$ belong to $\mathcal{K}$. The $\mathcal{A}(\mathcal{O})$ are supposed to act on a common Hilbert space $\mathcal{H}$. Since the family $\mathcal{K}$ is a direct set, the map $\mathcal{O} \rightarrow \mathcal{A}(\mathcal{O})$ is indeed a net and the quasilocal $C^{*}$-algebra $\mathcal{A}_{0}$ is defined as the direct limit of the local algebras. The algebras associated with general open regions in $M$ are defined by additivity.

Now we describe the conformal covariance assumption. As already explained, the group $\mathcal{C}$ acts locally on $M$. We observe that, since double cones are precompact, using the notation of formula (1.1) there exists an open neighborhood $V_{\mathcal{O}}$ of of the identity in $\tilde{\mathcal{C}}$ such that $V_{\mathcal{O}} \times \mathcal{O} \subset W$, and therefore the elements in $V_{\mathcal{O}}$ give rise to diffeomorphisms of $\mathcal{O}$ into $M$.

We assume that $\mathcal{C}$ acts locally by covariant automorphisms of the pre-cosheaf $\mathcal{A}$, namely, for any $\mathcal{O} \in \mathcal{K}$ there is a weakly continuous map from the open set $V_{\mathcal{O}}$ to the set $\operatorname{Iso}\left(\mathcal{A}(\mathcal{O}), \mathcal{A}_{0}\right)$ of isomorphisms of $\mathcal{A}(\mathcal{O})$ into $\mathcal{A}_{0}$,

$$
g \rightarrow \alpha_{g}^{\mathcal{O}}
$$

with the following properties.

If $\mathcal{O}_{1} \subset \mathcal{O}_{2}$ are double cones,

$$
\left.\alpha_{g}^{\mathcal{O}_{2}}\right|_{\mathcal{A}\left(\mathcal{O}_{1}\right)}=\alpha_{g}^{\mathcal{O}_{1}}
$$

Because of the preceding property, and since double cones form a net, we may drop the superscript which specify the region, $\alpha_{g} \equiv \alpha_{g}^{\mathcal{O}}$.

The map $g \rightarrow \alpha_{g}$ is a local action, i.e.

$$
\alpha_{h g}=\alpha_{h} \cdot \alpha_{g}
$$

when it makes sense.

The local action is covariant, i.e.

$$
\alpha_{g} \mathcal{A}(\mathcal{O})=\mathcal{A}(g \mathcal{O})
$$

Finally we assume the existence of a local unitary representation $U$ of $\mathcal{C}$ and of a $U$-invariant vector $\Omega$, cyclic for $\cup \mathcal{A}(\mathcal{O})$, such that

$$
U(g) A \Omega \equiv \alpha_{g}(A) \Omega \quad A \in \mathcal{A}(\mathcal{O}), \quad g \in V_{\mathcal{O}}
$$


The generators of the (local) one-parameter subgroups are well-defined selfadjoint operators on $\mathcal{H}$. The energy-momentum is assumed to be positive.

While the usual energy $H$ corresopnds to the Lie algebra generator $h$ of the time translations, the conformal energy $K$ corresponds to the Lie algebra element

$$
k \equiv h+\rho h \rho
$$

where $\rho h \rho$ is the adjoint action of $\rho$ on $h$. It is clear that if $H$ is positive, $K$ is the sum of positive operators, and therefore is positive too. The converse is also true, and can be checked on explicit realizations of the irreducible positive-energy unitary representation of $\mathcal{C}[19]$.

By the Reeh-Schlieder theorem $\Omega$ is cyclic and separating for the algebras $\mathcal{A}(\mathcal{O})$ and $U$ can be defined directly from (1.6). Now we show how the pre-cosheaf $\mathcal{A}$ may be canonically extended to the superworld $\tilde{M}$.

We observe that the map $g \rightarrow U(g)$ may be considered as a local unitary representation of the universal covering $\tilde{\mathcal{C}}$, where $g \in \tilde{V}_{\mathcal{O}}$, the identity component of the pre-image of $V_{\mathcal{O}}$ under the covering map. Then, since $\tilde{\mathcal{C}}$ is simply connected, $U$ extends to a global unitary representations of $\tilde{\mathcal{C}}$ on $\mathcal{H}$.

1.9 Lemma. If $\mathcal{O} \in \mathcal{K}, g \in \tilde{\mathcal{C}}$ and $g \mathcal{O}=\mathcal{O}$ then $U(g) \mathcal{A}(\mathcal{O}) U(g)^{*}=\mathcal{A}(\mathcal{O})$.

Proof We take $x \in \mathcal{O}$, then $g x \in \mathcal{O}$ and since the connected component of the stabilizer of $\mathcal{O}$ acts transitively on $\mathcal{O}$ (see Proposition 1.3) we find $h \in \tilde{\mathcal{C}}(\mathcal{O})_{0}$ such that $h^{-1} g x=x$. Then $h^{-1} g \in \tilde{\mathcal{C}}_{x}$, which is connected by construction (cf. the proof of Proposition 1.2). Therefore we may find a one-parameter family $l(t) \in \tilde{\mathcal{C}}_{x}$ such that $l(0)=i d$ and $l(1)=h^{-1} g$, and there exists a neighborhood $B_{x} \in \tilde{\mathcal{K}}$ of $x$ such that $l(t) B_{x} \subset \mathcal{O}, t \in[0,1]$, hence the local covariance of $\alpha$ implies covariance for all $t \in[0,1]$, i.e.

$$
\alpha_{h^{-1} g} \mathcal{A}\left(B_{x}\right)=\mathcal{A}\left(h^{-1} g B_{x}\right) .
$$

Since $h \in \tilde{\mathcal{C}}(\mathcal{O})_{0}$, we may connect it with $i d$ staying inside $\tilde{\mathcal{C}}(\mathcal{O})_{0}$, and the same argument used before implies $\alpha_{h} \mathcal{A}(\mathcal{O})=\mathcal{A}(\mathcal{O})$. As a consequence,

$$
\alpha_{g} \mathcal{A}\left(B_{x}\right)=\alpha_{h} \alpha_{h^{-1} g} \mathcal{A}\left(B_{x}\right)=\mathcal{A}\left(g B_{x}\right) \subset \mathcal{A}(\mathcal{O})
$$

By additivity $\mathcal{A}(\mathcal{O})=\vee_{x \in \mathcal{O}} \mathcal{A}\left(B_{x}\right)$, therefore

$$
\alpha_{g}(\mathcal{A}(\mathcal{O}))=\bigvee_{x \in \mathcal{O}}\left(\mathcal{A}\left(g B_{x}\right)\right)=\mathcal{A}(g \mathcal{O}) .
$$


Since $\tilde{\mathcal{C}}$ acts transitively on $\tilde{\mathcal{K}}$, we may define

$$
\tilde{\mathcal{A}}(g \mathcal{O})=U(g) \mathcal{A}(\mathcal{O}) U(g)^{*}, \quad g \in \tilde{G}, \mathcal{O} \in \mathcal{K} .
$$

By Lemma 1.9, the map $\tilde{\mathcal{A}}$ is well defined on $\tilde{\mathcal{K}}$, and defines a pre-cosheaf of $C^{*}$-algebras on $\tilde{\mathcal{K}}$. By definition $\tilde{\mathcal{C}}$ acts globally covariantly on the pre-cosheaf $\tilde{\mathcal{A}}$. We observe that if $W \subset M$ is a wedge, then $\tilde{\mathcal{A}}(W)$ turns out to be weakly closed, while $\mathcal{A}(W)$ is not. However $\tilde{\mathcal{A}}(W)=\mathcal{A}(W)^{\prime \prime}$, therefore, since $\mathcal{A}(W)$ is defined by additivity and $\mathcal{A}$ is causal, $\tilde{\mathcal{A}}(W) \subset \tilde{\mathcal{A}}\left(W^{\prime}\right)^{\prime}$. Then, by covariance and transitivity on $\tilde{\mathcal{K}}$, causality holds

for $\tilde{\mathcal{A}}$. The additivity property for $\tilde{\mathcal{A}}$ follows by the analogous property of $\mathcal{A}$. Thus we have proven the following:

1.10 Proposition. The pre-cosheaf $\mathcal{A}$ extends to a unique, causal, additive precosheaf $\tilde{\mathcal{A}}$ on $\tilde{M}$ and the local action $\alpha$ canonically extends to a globally covariant action of the group $\tilde{\mathcal{C}}$

We have therefore shown the equivalence between the locally covariant picture, on the manifold $M$, and the globally covariant picture, on $\tilde{M}$, for conformally covariant field theories.

As it is explained in ([10], see also [14]) we may define a universal $C^{*}$-algebra $\tilde{\mathcal{A}}_{0}$, and the isomorphisms ad $U(g)$ extend to automorphisms $\tilde{\alpha}_{g}$ of $\tilde{\mathcal{A}}_{0}$. The $C^{*}$-algebra $\tilde{\mathcal{A}}_{0}$ is larger than $\mathcal{A}_{0}$ since it contains the von Neumann algebras associated with wedge regions, and it is not necessarily faithfully represented in the vacuum representation $[10]$.

Finally we mention that explicit models may live on finite coverings of $\bar{M}$ or even on $\bar{M}$ itself (see examples in Section 2). In the last part of the following section we shall illustrate with some examples this phenomenon.

\section{Duality property and the Bisognano-Wichman theorem for conformal theories.}

In this Section we prove that essential duality holds for an algebraic conformal field theory on the Minkowski space $M$, and duality for double cones (and conformally equivalent regions) holds for the corresponding pre-cosheaf extension on $\tilde{M}$. Moreover the modular unitary group of a region $\mathcal{O} \in \tilde{\mathcal{K}}$ coincides with a one-parameter subgroup of the conformal group, namely we have an algebraic derivation of Bisognano-Wichmann theorem in this case.

As was explained in the preceding section, the Minkowski space $M$ is embedded in a canonical way in the superworld $\tilde{M}$, and a (locally) conformally covariant precosheaf of von Neumann algebras on $M$ extends uniquely to a pre-cosheaf on $\tilde{M}$ which is globally covariant with respect to the universal covering $\tilde{\mathcal{C}}$ of the conformal group $\mathcal{C}$. 
Therefore, in the following, $M$ will always be thought as a submanifold of $\tilde{M}$, and $\mathcal{A}$ as a sub-pre-cosheaf of $\tilde{\mathcal{A}}$. We shall denote by $\mathcal{R}(\mathcal{O})$ the von Neumann algebra associated with $\mathcal{O}$, both when $\mathcal{O} \in \mathcal{K}$ and when $\mathcal{O} \in \tilde{\mathcal{K}}$

$$
\mathcal{R}(\mathcal{O})=\tilde{\mathcal{A}}(\mathcal{O})
$$

In two-dimensional space-time theories we make the further assumption that parity is implemented, i.e. there exists a selfadjoint unitary $U(P)$ such that

$$
U(P) \mathcal{R}(\mathcal{O}) U(P)=\mathcal{R}(P \mathcal{O})
$$

where $P$ is the change of sign of the space coordinate in $M$, or the corresponding transformation in $\tilde{M}$. In particular, for chiral theories, namely pre-cosheaves which split into a product of two pre-cosheaves on $S^{1}$, parity correspond to the flip automorphism of the tensor product.

We recall that an internal symmetry of the theory is an automorphism of the pre-cosheaf, i.e. a consistent family of automorphisms

$$
\gamma^{\mathcal{O}} \in \operatorname{Aut}(\mathcal{R}(\mathcal{O})), \quad \mathcal{O} \in \tilde{\mathcal{K}}
$$

2.1 Lemma. Let $\mathcal{O}$ be a region in $\tilde{\mathcal{K}}, \Delta_{\mathcal{O}}$ the modular operator for $(\mathcal{R}(\mathcal{O}), \Omega)$ and $U_{\mathcal{O}}(t)$ the unitaries associated with the transformations $\Lambda_{t}^{\mathcal{O}}$ defined in (1.6). Then

$$
z(t)=\Delta_{\mathcal{O}}^{i t} U_{\mathcal{O}}(-t)
$$

is a one-parameter unitary group that commutes with the unitary representation of $\tilde{\mathcal{C}}$, implements internal symmetries, and does not depend on the region $\mathcal{O}$.

Proof $d>2$. Let us consider the wedge $W_{1}$ and the equation

$$
\Delta_{W_{1}}^{i t} U(g) \Delta_{W_{1}}^{-i t}=U_{W_{1}}(t) U(g) U_{W_{1}}(-t)
$$

If $g$ is a conformal diffeomorphism which preserves $W_{1}$, then $g$ commutes with $\Lambda_{t}^{W_{1}}$. Moreover $U(g)$ implements an automorphism of $\mathcal{R}\left(W_{1}\right)$, hence, by Tomita-Takesaki theory (see e.g. [20]), it commute with $\Delta_{W_{1}}^{i t}$ and (2.1) holds. This is also the case when $g$ is a translation along the axes $x_{2}, \ldots, x_{d-1}$ or the trasformations $\pm R_{2}$ introduced in Lemma 1.5. By Borchers theorem [3], (2.1) holds if $g$ is a translation along $x_{0}$ or $x_{1}$, and therefore, by Lemma 1.6, for all elements of the conformal group. As a consequence, $z(t)=\Delta_{W_{1}}^{i t} U(-t)$ commutes with $U(\tilde{\mathcal{C}})$ and therefore, since it preserves $\mathcal{R}\left(W_{1}\right)$, it preserves $\mathcal{R}(\mathcal{O})$ for any region $\mathcal{O} \in \tilde{\mathcal{K}}$. Hence $z(t)$ is an internal symmetry 
and commutes with the modular groups of all regions in $\tilde{\mathcal{K}}$. The independence from the region follows by the mentioned commutation relations.

If $d=2$, the proof goes on as before provided we substitute $R_{2}$ with $R_{1} P$.

If $d=1$ (i.e. on $S^{1}$ ), we consider the tensor product of $\tilde{\mathcal{A}}_{0}$ with itself, and we get a chiral two-dimensional theory. Then formula (2.1) holds for this theory, hence for the original one-dimensional theory.

2.2 Corollary. The given unitary representation $\{U(g): g \in \tilde{\mathcal{C}}\}$ is the unique representation of the conformal group which implements a covariant action on the pre-cosheaf $\tilde{\mathcal{A}}$ and preserves the vacuum vector.

Proof By Lemma 2.1 we get the equality of the multiplicative commutators

$$
\left[\Delta_{\mathcal{O}_{1}}^{i t}, \Delta_{\mathcal{O}_{2}}^{i s}\right]=\left[U\left(\Lambda_{t}^{\mathcal{O}_{1}}\right), U\left(\Lambda_{s}^{\mathcal{O}_{2}}\right)\right] .
$$

Therefore the representation $U$ is intrinsic for $g \in[\tilde{\mathcal{C}}, \tilde{\mathcal{C}}]$, since it it determined by the modular group. Since $\tilde{\mathcal{C}}$ is perfect (Proposition 1.4), $U$ is completely determined by the modular operators, hence it is unique.

2.3 Theorem. Let $\mathcal{A}$ be a conformally covariant pre-cosheaf on $M, \tilde{A}$ the corresponding pre-cosheaf on $\tilde{M}$. Then:

(i) Essential duality holds for the pre-cosheaf $\mathcal{A}$, and duality holds for the pre-cosheaf $\tilde{\mathcal{A}}$.

(ii) If $\mathcal{O}$ is a region in $\tilde{\mathcal{K}}, \Delta_{\mathcal{O}}$ the modular operator for $(\mathcal{R}(\mathcal{O}), \Omega)$ and $U_{\mathcal{O}}(t)$ the unitary associated with $\Lambda_{t}^{\mathcal{O}}$, then

$$
\Delta_{\mathcal{O}}^{i t}=U_{\mathcal{O}}(t)
$$

Proof (i) Let us consider the wedge $W_{1}$. By causality, $\mathcal{R}\left(W_{1}^{\prime}\right)$ is a subalgebra of $\mathcal{R}\left(W_{1}\right)^{\prime}$, and is globally stable under the action of the modular group of $\mathcal{R}\left(W_{1}\right)$ because the latter acts geometrically by Proposition 2.1. Therefore, by Tomita-Takesaki theory and the cyclicity of the vacuum, it coincides with $\mathcal{R}\left(W_{1}\right)^{\prime}$. Since the Poincaré group has a global causally-preserving action on $M$ the relation

$$
\mathcal{R}\left(W^{\prime}\right)=\mathcal{R}(W)^{\prime}
$$

holds for each wedge $W$, i.e. essential duality holds for the pre-cosheaf $\mathcal{A}$. Since $\tilde{\mathcal{C}}$ has a global causally-preserving action on $\tilde{M}$ the relation

$$
\mathcal{R}\left(\mathcal{O}^{\prime}\right)=\mathcal{R}(\mathcal{O})^{\prime}
$$


holds for any region $\mathcal{O} \in \mathcal{K}$, i.e. duality holds for the pre-cosheaf $\tilde{\mathcal{A}}$.

(ii) We have to show that $z(t)$ in Lemma 2.1 vanishes. Let us consider one of the conformal transformations $\pm R_{1}$ introduced in Proposition 1.5. By definition, $R_{1} W_{1}=$ $W_{1}^{\prime}$. Then, by essential duality,

$$
U\left(R_{1}\right) \Delta_{W_{1}} U\left(R_{1}\right)=\Delta_{W_{1}}^{-1}
$$

Moreover,

$$
U\left(R_{1}\right) U_{W_{1}}(t) U\left(R_{1}\right)=U\left(R_{1} \Lambda_{t}^{W_{1}} R_{1}\right)=U_{W_{1}}(-t)
$$

Therefore,

$$
z(t)=U\left(R_{1}\right) z(t) U\left(R_{1}\right)=U\left(R_{1}\right) \Delta_{W_{1}}^{i t} U_{W_{1}}(-t) U\left(R_{1}\right)=z(-t)
$$

hence $z(t)=I$.

Now we show that in even dimensions there exists a canonical antiunitary $\Theta$ which implements the PCT transformation on $\tilde{M}$, i.e. implements a conformal transformation of $\tilde{M}$ that restricts to change of sign of all coordinates on $M$ :

$$
\Theta \mathcal{R}(\mathcal{O}) \Theta=\mathcal{R}(\beta \mathcal{O}), \quad \mathcal{O} \in \mathcal{K}
$$

where $\beta x=-x, x \in M$. Such an antiunitary is not unique, since $\Theta V$ still implements a PCT whenever $V$ is a self-adjoint unitary which implements a internal symmetry. A suitable (positivity) condition will fix a canonical choice for $\Theta$. We choose the wedge $W_{1}$ as in formula (2.1) and consider the unitary $S_{W_{1}}$ which corresponds to the change of sign of the coordinates $x_{2}, \ldots x_{d-1}$ (which is an element in the identity component of $\tilde{\mathcal{C}}$ when $d$ is even). Then, with each wedge $W$ in the family $\mathcal{W}_{0}$ of the Lorentz transformed regions of $W_{1}$, we associate the unitary $S_{W} \equiv U(g) S_{W_{1}} U\left(g^{-1}\right)$ where $g W_{1}=W, g \in \mathcal{L}_{+}^{\uparrow}$.

2.5 Theorem. The anti-unitary $\Theta=J_{W} S_{W}, W \in \mathcal{W}_{0}$, implements a PCT transformation and does not depend on the choice of the wedge $W \in \mathcal{W}_{0}$. It is the unique anti-unitary such that

$$
\Theta \mathcal{R}(\mathcal{O}) \Theta=\mathcal{R}(\beta \mathcal{O}) \quad \mathcal{O} \in \mathcal{K}
$$

and

$$
\left(\Omega, A \Theta S_{W} A \Omega\right) \geq 0 \quad \forall A \in \mathcal{R}(W), \quad W \in \mathcal{W}_{0}
$$

Proof The same arguments used in the proof of Theorem 2.1 imply that

$$
J_{W_{1}} U(g) J_{W_{1}}=U\left(r_{1} g r_{1}\right), \quad g \in \mathcal{C}
$$


where $J_{W_{1}}$ is the modular conjugation for the algebra $\mathcal{R}\left(W_{1}\right)$ and $r_{1}$ is the reflection with respect to the edge of the wedge. Since $J_{W_{1}} \mathcal{R}\left(W_{1}\right) J_{W_{1}}=\mathcal{R}\left(W_{1}^{\prime}\right)=\mathcal{R}\left(r_{1} W_{1}\right)$ we get

$$
J_{W_{1}} \mathcal{R}(\mathcal{O}) J_{W_{1}}=\mathcal{R}\left(r_{1} \mathcal{O}\right), \quad \forall \mathcal{O} \in \mathcal{K}
$$

Hence $J_{W_{1}} S_{W_{1}}$ implements the transformation $\beta$ defined by $\beta x=-x$. Therefore the map

$$
g \rightarrow \Theta U(\beta g \beta) \Theta \quad g \in \tilde{\mathcal{C}}
$$

is a covariant unitary representation of $\tilde{\mathcal{C}}$. By the uniqueness proved in Corollary 2.2, and since $\beta$ commutes with the Lorentz group $\mathcal{L}_{+}^{\uparrow}$ we get

$$
\Theta U(g) \Theta=U(g) \quad g \in \mathcal{L}_{+}^{\uparrow}
$$

The previous commutation relation implies that $\Theta$ does not depend on $W$ :

in fact given two wedges $W_{1}, W_{2}$ we can find an element $g \in \mathcal{L}_{+}^{\uparrow}$ such that $g W_{1}=W_{2}$, therefore we have

$$
J_{W_{1}} S_{W_{1}}=U(g) J_{W_{2}} U(g) U(g)^{*} S_{W_{2}} U(g)^{*}=J_{W_{2}} S_{W_{2}} .
$$

Since $\Theta$ implements the transformation $\beta, \Theta S_{W}$ is an antilinear conjugation which maps $W$ in $W^{\prime}$, hence, by essential duality, $\Theta S_{W} \mathcal{R}(W) \Theta S_{W}=\mathcal{R}(W)^{\prime}$. Therefore condition (2.2) is the characterization of the modular conjugation $J_{W}[20]$, and the uniqueness follows.

We observe that in odd dimensions we may implement a PCT transformation up to a change of sign of one space coordinate. Therefore a complete PCT is implemented if and only if such a change of sign is unitarily implemented.

2.6 Remark. The split property [8] holds automatically for a conformal pre-cosheaf on $\left(S^{d-1} \times S^{1}\right) / \mathbf{Z}_{2}$, where it is equivalent to the distal split property [8] by conformal invariance, provided the conformal Hamiltonian $K$ (that has spectrum equal to $\mathbf{N}$ ) has the multiplicity of its eigenvalues growing at most exponentially. Indeed $e^{-\beta K}$ is a trace-class operator (for $\beta$ large enough) and nuclearity holds [5].

2.7 Corollary. With the assumptions of the previous remark, any local algebra $\mathcal{R}(\mathcal{O}), \quad \mathcal{O} \in \tilde{\mathcal{K}}$, is the unique injective factor of type $I I I_{1}$.

Proof Immediate, see [16].

Examples derived from free fields. We illustrate the present setting by some examples given by the net $\mathcal{A}$ of local algebras associated with the free massless scalar 
field on the $d$-dimensional Minkowski space discussed in [15], with a reinterpretation of the duality behavior.

Recall that the group of conformal time translations (i.e. the subgroup generated by the conformal energy $K$ ) has period $2 \pi$, i.e. the operator $e^{2 \pi i K}$ acts as the identity on $\tilde{\mathcal{A}}$. The $\mathbf{Z}$-component of the center of $\tilde{\mathcal{C}}$ acts as an "helicoidal shift" on $\tilde{M}$ and that the $2 \pi \mathbf{Z}$ time translations correspond to the even part of the $\mathbf{Z}$-component of the center of $\tilde{\mathcal{C}}$. Therefore all even copies of $M$ may be identified, and all odd copies of $M$ may be identified, hence free massless scalar fields lives on the compactification $\bar{M}$ or on its 2-fold covering $\bar{M}_{2}$. In the following, if $\mathcal{O}$ is a region in $M$, we shall indicate with $\mathcal{O}^{\prime}$ its space-like complement in $M$, with $\mathcal{O}^{t}$ its time-like complement in $M$, and with $\mathcal{O}^{c}$ its causal complement in $\bar{M}$, resp. $\bar{M}_{2}$.

- $d$ even. Since the relativistic ray inversion $\rho$ is unitarily implemented, the theory lives on $\bar{M}$. The space-like complement $\mathcal{O}^{\prime}$ and the time-like complement $\mathcal{O}^{t}$ of a double cone $\mathcal{O} \in M$ form a connected region $\mathcal{O}^{c}$ in $\bar{M}$, the causal complement of $\mathcal{O}$ in $\bar{M}$. The duality property in $\bar{M}$ means

$$
\mathcal{R}(\mathcal{O})^{\prime}=\mathcal{R}\left(\mathcal{O}^{c}\right)
$$

Since $\mathcal{R}\left(\mathcal{O}^{\prime}\right)$ and $\mathcal{R}\left(\mathcal{O}^{t}\right)$ are subalgebras of $\mathcal{R}\left(\mathcal{O}^{c}\right)$, space-like and time-like commutativity hold for the pre-cosheaf $\mathcal{A}$. Moreover $\mathcal{R}\left(\mathcal{O}^{\prime}\right)=\mathcal{R}\left(\mathcal{O}^{c}\right)$, therefore Haag duality holds in $M$. If $d>2, \mathcal{R}\left(O^{t}\right) \neq \mathcal{R}\left(\mathcal{O}^{c}\right)$, i.e. time-like duality does not hold in $M$ [15]. If $d=2, \mathcal{R}\left(\mathcal{O}^{t}\right)=\mathcal{R}\left(\mathcal{O}^{c}\right)$, i.e. time-like duality holds in $M$.

- $d$ odd $(d \neq 1)$. Since two different liftings of $\rho$ in $\tilde{\mathcal{C}}$ are unitarily implemented, the theory lives on the 2 -fold covering $\bar{M}_{2}$. The product of the two liftings of the relativistic ray inversion is the transformation $\mathcal{O} \rightarrow \tilde{\mathcal{O}}$ which maps a region in one of the copies of $M$ in $\bar{M}_{2}$ onto the corresponding region in the other copy. The causal complement in $\bar{M}_{2}$ of a double cone $\mathcal{O} \in M$ is the region

$$
\mathcal{O}^{c}=\mathcal{O}^{\prime} \cup \tilde{\mathcal{O}}^{t}
$$

Since

$$
\mathcal{R}\left(\mathcal{O}^{\prime}\right)=\mathcal{R}\left(\mathcal{O}^{c}\right)
$$

then Haag duality follows by the duality property for the pre-cosheaf $\tilde{\mathcal{A}}$. The "twisted time-like commutativity" in [15] is the inclusion

$$
\mathcal{R}\left(\tilde{\mathcal{O}}^{t}\right) \subset \mathcal{R}(\mathcal{O})^{\prime}
$$

i.e. the algebra associated to the twisted time-complement $\tilde{\mathcal{O}}^{t}$ commutes with $\mathcal{R}(\mathcal{O})$. 
- $d=1$. Fix $n>2$ and let $\mathcal{R}(\mathcal{O})$ be the net on the $n$-dimensional Minkowski space described above. Then set

$$
\mathcal{A}_{n}(I)=\mathcal{R}(\mathcal{O})
$$

where $I \subset \mathbf{R}$ is an interval of the time axis and $\mathcal{O}$ is the double cone obtained by causal completion from $I$.

If $n$ is even then the $\mathcal{A}_{n}(I)$ give a conformal net on $\mathbf{R}$ that extends to a Möbius covariant positive energy pre-cosheaf on $S^{1}$. $\mathcal{A}_{n}$ satisfies duality on $S^{1}$. However $\mathcal{A}_{n}$ satisfies duality on $\mathbf{R}$ iff $n=2$.

If $n$ is odd, $\mathcal{A}_{n}$ extends to a Möbius covariant positive energy pre-cosheaf on the disjoint union $S^{1} \sqcup S^{1}$. If $I$ is an interval in one of the copies of $S^{1}$, we denote by $\tilde{I}$ the corresponding interval in the other copy of $S^{1}$. Then duality holds in the form

$$
\mathcal{A}_{n}(I)^{\prime}=\mathcal{A}_{n}\left(\tilde{I}^{\prime}\right)
$$

where $I^{\prime}$ is the interior of the complement of $I$.

Finally we notice that, with the previous notations, the tensor product

$$
\mathcal{A}_{4} \otimes \mathcal{A}_{4}
$$

is a conformally covariant pre-cosheaf on $\mathbf{R}^{2}$ such that Haag duality does not hold.

\section{Further results for Poincaré covariant theories}

In this section we consider Poincaré covariant Quantum Field Theories in $d>2$ dimensions, namely a pre-cosheaf of von Neumann-algebras $\mathcal{O} \rightarrow \mathcal{R}(\mathcal{O})$ where $\mathcal{O}$ is in the family $\mathcal{K}$ of double cones in the Minkowski space $M$ with the usual causality and additivity properties (see Section 1).

The quasi-local $C^{*}$-algebra $\mathcal{A}_{0}$ generated by the local algebras is supposed to act in the vacuum representation as usual [9].

The Poincaré group $\mathcal{P}_{+}^{\uparrow}$ acts by covariant automorphisms on $\mathcal{A}_{0}$ and positivity of the energy-momentum is required.

The pre-cosheaf is extended by additivity to general open regions in $M$. The weak closure of the algebra associated with an unbounded region $\mathcal{O}$ will be denoted by $\mathcal{R}(\mathcal{O})$.

We shall make two main assumptions in this Section:

(a) Given any wedge region $W$, the modular unitaries $\Delta_{W}^{i t}$ of $\mathcal{R}(W)$ act geometrically on the pre-cosheaf $\mathcal{A}$,

$$
\Delta_{W}^{i t} \mathcal{R}(\mathcal{O}) \Delta_{W}^{-i t}=\mathcal{R}\left(\Lambda_{t}^{W} \mathcal{O}\right), \quad \mathcal{O} \in \mathcal{K}, \quad \forall W \in \mathcal{W}
$$

where $\Lambda_{t}^{W}$ is the one-parameter group of diffeomorphisms defined in 1.6, and $\mathcal{W}$ is the family of all wedge regions in $M$. 
(b) Distal split property holds, i.e there exist two regions $\mathcal{O}_{1} \subset \mathcal{O}_{2}^{\prime}$ in $M$ such that $\mathcal{R}\left(\mathcal{O}_{1}\right)$ and $\mathcal{R}\left(\mathcal{O}_{2}\right)$ generates a $W^{*}$-tensor product.

3.1 Theorem. If distal split property holds, there is only one covariant unitary representation of the Poincaré group on $\mathcal{H}$ leaving the vacuum vector invariant.

Proof Distal split property implies that the group of internal symmetries $\mathcal{G}$ is compact and commutes with any action of the Poincaré group by automorphisms [8]. Then if two unitary covariant representations $U, V$ of $\mathcal{P}_{+}^{\uparrow}$ exist, $\operatorname{ad} U(g) V\left(g^{-1}\right)$ belong to $\mathcal{G}$, and therefore gives rise to an action of $\mathcal{P}_{+}^{\uparrow}$ in $\mathcal{G}$. Since $\mathcal{P}_{+}^{\uparrow}$ has no non-trivial finitedimensional representations, and $\mathcal{G}$ is compact, such a representation is trivial, i.e. $\operatorname{ad} U(g) V\left(g^{-1}\right)=i d$. Then $U(g) V\left(g^{-1}\right)$ is a one-dimensional representation of $\mathcal{P}_{+}^{\uparrow}$, and, repeating the preceding argument, $U(g) V\left(g^{-1}\right)=1$.

3.2 Lemma. Let $W$ be a wedge in $\mathcal{W}, \Delta_{W}$ the modular operator for $(\mathcal{R}(W), \Omega)$ and $U_{W}(t)$ the unitary associated with $\Lambda_{t}^{W}$. Then, if assumptions $(a)$ and $(b)$ hold

$$
z(t)=\Delta_{W}^{i t} U_{W}(-t)
$$

is a one-parameter group which commutes with the unitary representation of $\mathcal{P}_{+}^{\uparrow}$, implements internal symmetries and does not depend on the wedge $W$.

Proof By assumption $(a)$, the unitaries

$$
z_{t}^{W}=\Delta_{W}^{-i t} U_{W}(t) \quad, \quad W \in \mathcal{W}, \quad t \in \mathbf{R}
$$

implement internal symmetries, and therefore commute with the modular groups of any local algebra.

Moreover, if we fix $W_{0} \in \mathcal{W}$ and $W_{1}$ is any other wedge, we can find an element $g \in \mathcal{P}_{+}^{\uparrow}$ which maps $W_{1}$ onto $W_{0}$, and therefore

$$
z^{W_{1}}(t)=U\left(g^{-1}\right) z^{W_{0}}(t) U(g) \quad t \in \mathbf{R}
$$

Since assumption $(b)$ implies that the internal symmetries commute with any action of $\mathcal{P}_{+}^{\uparrow}$ by automorphisms [8], the unitary $z^{W_{1}}(t) z^{W_{0}}(-t) \in \mathbf{C}$. Since it preserves the vacuum vector, then $z_{t} \equiv z_{t}^{W_{0}}$ is independent from $W_{0}$.

Finally, the group property of $\{z(t), t \in \mathbf{R}\}$ is easily checked using the mentioned commutation relations.

3.3 Theorem. Let $\mathcal{A}$ be a local pre-cosheaf on $M$ satisfying assumption $(a)$. Then the following holds: 
(i) The pre-cosheaf $\mathcal{A}$ satisfies essential duality, i.e.

$$
\mathcal{R}(W)^{\prime}=\mathcal{R}\left(W^{\prime}\right)
$$

for each wedge region $W$.

(ii) If distal split property holds and $d>2$, then

$$
\Delta_{W}^{i t}=U_{W}(t)
$$

where $\Delta_{W}$ is the modular operator for $(\mathcal{R}(W), \Omega)$ and $U_{W}(t)$ is the unitary representation of the boosts which preserve $W$. The same result holds when $d=2$, provided that the parity transformation is unitarily implemented.

Proof The proof of part $(i)$ is identical to the proof of Theorem 2.3, part $(i)$. Since Lemma 3.4 holds, the proof of $(i i)$ is analogous to the proof of Theorem 2.3, part $(i i)$, provided that $R_{i}$ is replaced by a Poincaré transformation mapping a wedge $W$ onto $W^{\prime}$. If $d>2$ we may choose a rotation, if $d=2$ we use the parity transformation.

We conclude this section with an example of a non-split net where $(i i)$ of Theorem 3.3 does not hold, namely $z(t)$ is non trivial.

Such a construction was mentioned to us by D. Buchholz in a different context. Let $\mathcal{A}$ be a Poincaré covariant net on the $d+1$-dimensional Minkowski space $M_{d+1}$ which satisfies the Bisognano-Wichmann theorem. Define a net $\mathcal{B}$ on the $d$-dimensional Minkowski space $M_{d}$ by

$$
\mathcal{B}(\mathcal{O})=\mathcal{A}\left(\pi^{-1} \mathcal{O}\right) \quad \mathcal{O} \subset M_{d}
$$

where $\pi: M_{d+1} \rightarrow M_{d}$ is the projection parallel to the $(d+1)$-th coordinate.

Let $W_{1}$ be a wedge in $M_{d}, z(t)$ the translation along the $(d+1)$ axis. Then $z(t)$ implements a one-parameter group of internal symmetries of $\mathcal{B}$ which commutes with the action of the Poincaré group on $M_{d}$, hence

$$
U_{W}^{\prime}(t) \equiv z(t) U_{W}(t)
$$

determines a new $d$-dimensional representation of $\mathcal{P}_{+}^{\uparrow}$ that violates the BisognanoWichmann theorem. 


\section{References}

[1] Araki H., "A lattice of von Neumann algebras associated with the quantum field theory of a free Bose field", J. Math. Phys. 4 (1963), 1343-1362.

[2] Bisognano J., Wichmann E. , "On the duality condition for a Hermitian scalar field', J. Math. Phys. 16 (1975), 985-1007

[3] Borchers H.J., "The CPT theorem in two-dimensional theories of local observables", Comm. Math Phys. 143 (1992), 315-332

[4] Buchholz D., "On the structure of local quantum fields with non-trivial interaction", in: Proc. of the Int. Conf. on Operator Algebras, Ideals and their Applications in Theoretical Physics, Baumgärtel, Lassner, Pietsch, Uhlmann, (eds.) pp. 146-153. Leipzig: Teubner Verlagsgesellschaft 1978.

[5] Buchholz D., D'Antoni C., private communication.

[6] Buchholz D., Schulz-Mirbach H. , "Haag duality in conformal quantum field theory", Rev. Math. Phys. 2 (1990) 105.

[7] Dirac, P.A.M., "Wave equations in conformal space", Ann. Math. 37 (1936) 429.

[8] Doplicher S., Longo R. , "Standard and split inclusions of von Neumann algebras", Invent. Math. 73 (1984), 493.

[9] Haag R., "Local Quantum Physics", Springer Verlag, Berlin Heidelberg 1992.

[10] Fredenhagen K., "Generalization of the theory of superselection sectors", in The algebraic Theory of Superselection Sectors, D. Kastler ed., World Scientific, Singapore 1990.

[11] Fredenhagen, K., Jörss M., in preparation, see: Jörss M., On the existence of pointlike localized fields in conformally invariant quantum physics", Desy Preprint.

[12] Gabbiani F., Fröhlich J., "Operator algebras and Conformal Field Theory", preprint EHT Zürich, 1992.

[13] Gilman L., Jerison M. , "Rings of continuous functions" Springer-Verlag, New York Heidelberg Berlin 1976.

[14] Guido D., Longo R., "Relativistic invariance and charge conjugation in quantum field theory", Comm. Math. Phys. 148 (1992), 521-551.

[15] Hislop P., Longo R., "Modular structure of the local observables associated with the free massless scalar field theory", Comm. Math. Phys. 84 (1982), 84.

[16] Longo R., "Algebraic and modular structure of von Neumann algebra of physics", Proc. of Symposia in Pure Mathematics 38 (1982), part 2, 551-566.

[17] Mack G., Lüscher M., "Global conformal invariance in quantum field theory", Comm. Math. Phys. 41 (1975) 203.

[18] Postnikov, "Leçons de geometrie. Groupes et algebres de Lie, Éditios Mir, Moscou, 1985. 
[19] Segal I. E., "Causally oriented manifolds and groups", Bull. Amer. Math. Soc. 77 (1971) 958.

[20] Strătilă S., Zsido L. , Lectures on von Neumann algebras, Abacus press, England 1979.

[21] Streater R.F., Wightman A.S. , PCT, spin and statistics, and all that, Addison Wesley, Reading, MA 1989

[22] Todorov I. T., Mintchev M. C., Petkova V. B., Conformal invariance in quantum field theory, Publ. Scuola Normale Superiore, Pisa, 1978.

[23] Weyl H., Space-Time-Matter, Dover Pubblications Inc., 1950. references4-dim 